\title{
MODELS FOR NUCLEI OF PLANETARY NEBULAE AND FOR ULTRAVIOLET STARS
}

\author{
J. KATZ, R. MALONE, and E. E. SALPETER \\ Cornell University, Ithaca, N.Y., U.S.A.
}

\begin{abstract}
A series of stellar models were evolved, all with a total mass of $0.65 M_{\odot}$, an initial carbon-oxygen core of mass $0.60 M_{\odot}$, an intermediate helium mantle and an outer hydrogen-rich envelope with mass varying from case to case. Although the most hydrogen-rich cases resulted in red giants, cases with $\lesssim 0.01 M_{\odot}$ in the hydrogen envelope evolved at high surface temperature. The early stages of development of these models are similar to observed central stars of planetary nebulae. The later stages (when the nebula should be very thin optically) still have a high luminosity; the relevance to 'ultraviolet stars' will be discussed.
\end{abstract}

\section{DISCUSSION}

Demarque: Did you encounter any hydrogen shell flash?

Salpeter: Yes, some of these violent outward excursions were in fact hydrogen shell flashes. I should have said also that in the models which starts with a fair bit of hydrogen, roughly half of that hydrogen already burned before reaching that steady helium looping. Not too much more of the hydrogen was burned during the looping, much of it then burned later on, in shell flashes and steadily, with flashes going back and forth between the hydrogen and helium. Whatever you can imagine, it all happened.

Demarque: Do you really expect to see planetaries in large numbers in globular clusters? The only one that is known according to Peimbert has a mass in the nebula of the order of 1 hundredth of a solar mass. Now if horizontal branch stars have masses of the order of $6 / 10$ ths of a solar mass, they don't have much to eject, do they, and maybe they just bypass this phase; maybe one should expect to see them only in the old disc population where the masses are higher.

Salpeter: I quite agree that that's at least a possibility and even if Cahn and Kaler are right for population I, one has no way of knowing whether one should or should not expect them in globular clusters. It may even be that planetary nebulae are rare in globular clusters because of the lack of $\mathrm{H}$ shells but these ultraviolet stars are present. Incidently, I forgot to mention another indirect datum on this; Frank Kerr just earlier this week mentioned that searches for neutral and for ionised hydrogen in globular clusters have been made. Nothing has been found down to quite a low level. On the other hand, if anything I've said here is at all correct, that is not at all surprising because these ultraviolet stars, even after the nebula has become optically thin, still keep on driving the nebula out and so the velocities one gets (especially if some dust is present in the nebulae) are up to about $100 \mathrm{~km} \mathrm{~s}^{-1}$ and will have no difficulty escaping, so one would not predict theoretically that there should be hydrogen visible in globular clusters.

Faulkner: Are you now rejecting the correlation between observed nuclear properties as obtained from the Zanstra temperature and the Shklovski luminosities and the radius of the nebular shell which was the basis for assigning a several times $10^{4} \mathrm{yr}$ time scale on planetary nuclear evolution, in favour of this 10 times shorter scale?

Salpeter: Well I'm not quite sure whether I am or not, I'm at least forshadowing the rejection and that was in part what my last slide was for. The selection effect might mimic the horseshoe curve which might make you think that you already are going down into the white dwarf region but still at a redder colour. The other feature that looks a little bit like a hook are these loopings, that's why I emphasize the loopings. It might also be that some early planetary nebulae are still in a transient stage and that for some reason it's this part of the helium burning loops that are most prominent.

Iben: Could you elucidate once more the manner in which you make the hydrogen invisible? Before 
doing so, let me give you some numbers: if mass is lost from stars in the giant phase before becoming horizontal branch stars, to the tune of about $2 / 10$ ths $M_{\odot}$ per star and if the ejection velocities are larger than the escape velocity from the cluster, then you might expect something like a solar mass of hydrogen in the vicinity of the 10 parsec confines of the cluster. Now Kerr's limit is something like a solar mass or less, of neutral hydrogen and the limits on ionised hydrogen are something like $15 M_{\odot}$ and ultraviolet radiation will certainly turn that neutral hydrogen into ionised $\mathbf{H}$; I think there's enough ultraviolet from the very blue horizontal branch stars to do the job.

Salpeter: Assume that (unlike what Demarque said) there are planetary nebulae in globular clusters and that they provide the main mass loss: Even then these planetary nebulae (the central stars themselves and the ultraviolet stars that they become) are enough so that the hydrogen that escapes from the planetary nebula never even recombines and gets speeded up from $20 \mathrm{~km} \mathrm{~s}^{-1}$ up to about $100 \mathrm{~km} \mathrm{~s}^{-1}$ as it goes out. So therefore you never would see any neutral hydrogen at all, and you would only have maybe a dozen or so of those shells still within the confines of the globular cluster at any one time. $\left(\ll 15 M_{\odot}\right.$ in ionised gas).

Iben: I see; do you make the mass that comes off the planetary invisible?

Salpeter: Immediately, yes.

Webster: When deriving this evolutionary track, Harman and Seaton calculated the optical depth of the nebulae to radiation past the Lyman limit and found that the boundary between optically thick and thin nebulae was on the low temperature edge of the track. I think it is unlikely, for this reason, that the high temperature hook is the particular optical depth selection effect that you are suggesting.

Salpeter: Well, it may not be possible; but on the other hand the stage where the luminosity of the star ceases to matter for the nebula luminosity depends on the luminosity of the star and these excursions in luminosity are pretty big. I really haven't looked at the effect quantitatively. 